\title{
Mitochondrial genome sequencing helps show the evolutionary mechanism of mitochondrial genome formation in Brassica
}

\author{
Shengxin Chang ${ }^{1}$, Tiantian Yang ${ }^{1}$, Tongqing Du', Yongjuan Huang ${ }^{1}$, Jianmei Chen ${ }^{1}$, Jiyong Yan², Jianbo He ${ }^{1}$ and
} Rongzhan Guan ${ }^{1 *}$

\begin{abstract}
Background: Angiosperm mitochondrial genomes are more complex than those of other organisms. Analyses of the mitochondrial genome sequences of at least 11 angiosperm species have showed several common properties; these cannot easily explain, however, how the diverse mitotypes evolved within each genus or species. We analyzed the evolutionary relationships of Brassica mitotypes by sequencing.

Results: We sequenced the mitotypes of cam (Brassica rapa), ole (B. oleracea), jun (B. juncea), and car (B. carinata) and analyzed them together with two previously sequenced mitotypes of B. napus (pol and nap). The sizes of whole single circular genomes of cam, jun, ole, and car are 219,747 bp, 219,766 bp, 360,271 bp, and 232,241 bp, respectively. The mitochondrial genome of ole is largest as a resulting of the duplication of a $141.8 \mathrm{~kb}$ segment. The jun mitotype is the result of an inherited cam mitotype, and pol is also derived from the cam mitotype with evolutionary modifications. Genes with known functions are conserved in all mitotypes, but clear variation in open reading frames (ORFs) with unknown functions among the six mitotypes was observed. Sequence relationship analysis showed that there has been genome compaction and inheritance in the course of Brassica mitotype evolution.
\end{abstract}

Conclusions: We have sequenced four Brassica mitotypes, compared six Brassica mitotypes and suggested a mechanism for mitochondrial genome formation in Brassica, including evolutionary events such as inheritance, duplication, rearrangement, genome compaction, and mutation.

\section{Background}

Plant mitochondrial genomes are complex because they encode significantly more genes than do their fungal and animal counterparts. Investigations of the mitochondrial genome sequences of at least 13 angiosperm species, including Arabidopsis thaliana [1], Beta vulgaris [2], Oryza sativa [3-5], Brassica napus [6,7], Zea mays [8-10], Nicotiana tabacum [11], Triticum aestivum [12], Vitis vinifera [13], Citrullus lanatus and Cucurbita pepo [14], and Vigna radiata [15], together with physical mapping [16-18], have showed several properties of plant mitochondrial genomes, such as large size (200$2400 \mathrm{~kb})$, slow rates of evolutionary change,

\footnotetext{
* Correspondence: guanrzh@njau.edu.cn

'State Key Laboratory of Crop Genetics and Germplasm Enhancement,

Nanjing Agricultural University, Nanjing 210095, PR China

Full list of author information is available at the end of the article
}

incorporation of foreign DNA, a multipartite structure, and specific modes of gene expression (e.g. cis and trans splicing, RNA editing), etc [19]. These properties cannot easily explain how the diverse mitotypes evolved within each plant genus or species. To understand the evolutionary peculiarity of plant mitotypes, which are defined as mitochondrial genome types of which there can be more than one within one plant species or genus, more systematic sequences are needed. To date, no systematic sequences of one angiosperm genus with multiple species had been used to analyze the derivation of mitochondrial genome, and thus the mechanism underlying the peculiarity has not been revealed. Here, we selected Brassica as an evolutionary clade in which to analyze the evolutionary relationships of Brassica mitotypes by sequencing. 
Brassica contains six cultivated species that are very important for producing vegetables and oilseeds. The nuclear genomic relationships between the six species were showed by the U with cytogenetics approach [20], which has been popularly accepted as U's triangle theory (Figure 1). However, the cytoplasmic relationship between the six species needs to be further explored, although the entire sequences of two mitotypes of Brassica napus have been reported [6,7] with a focus on the mechanism of cytoplasmic male sterility (CMS), and mitochondrial DNA (mtDNA) of three Brassica species has been physically mapped [18]. Here, we report the sequence of the mitochondrial genomes of $B$. rapa (cam mitotype), B. oleracea (ole), B. juncea (jun), and B. carinata (car). Together with previously reported mitochondrial genome sequences of the pol and nap mitotypes in $B$. napus [6,7], we compared the six mitochondrial genome sequences to learn the mechanism of formation for Brassica mitochondrial genomes.

\section{Results}

\section{Brassica mitochondrial genomes}

Brassica comprises six cultivated species [21]: B. rapa (cam), B. oleracea (ole), B. napus, B. juncea (jun), B. nigra, and $B$. carinata (car). The sizes of entire single circular mitochondrial genomes of cam [GenBank: JF920285], jun [GenBank: JF920288], ole [GenBank: JF920286], and car [GenBank: JF920287] are 219,747 bp, $219,766 \mathrm{bp}, 360,271 \mathrm{bp}$, and 232,241 bp, respectively.

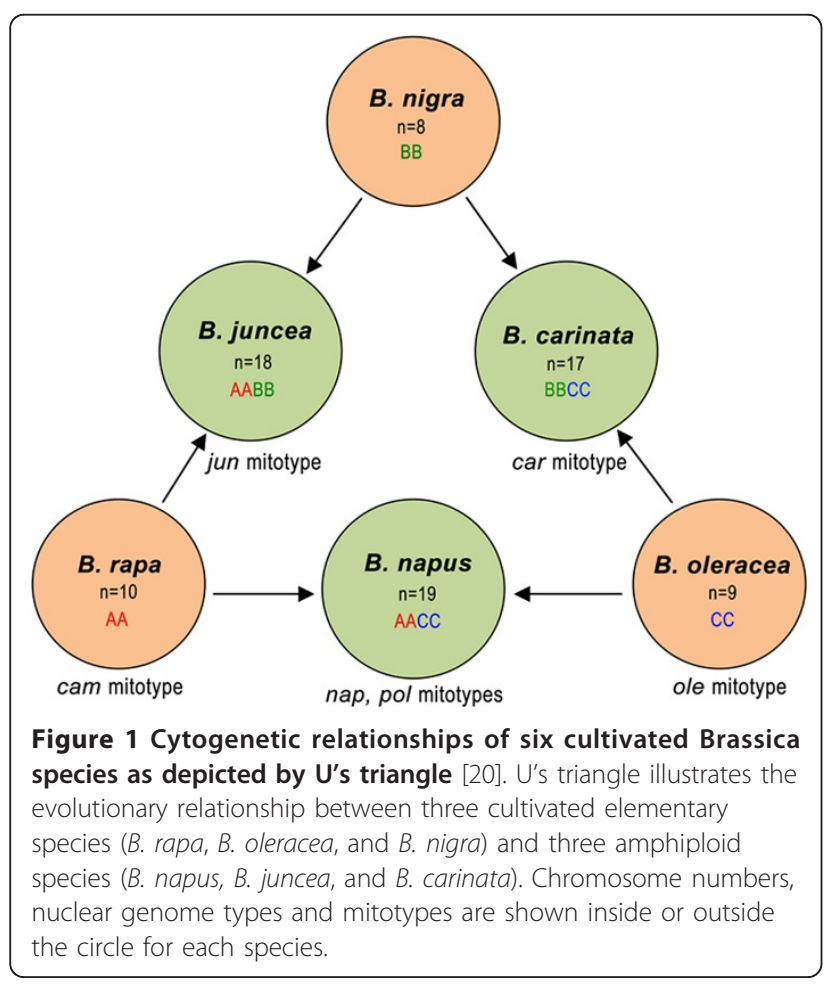

The mitochondrial genomes of car and ole are larger than these of cam, jun, pol, and nap. The ole mitotype is larger because of the duplication of a $141.8 \mathrm{~kb}$ segment. The $\mathrm{G}+\mathrm{C}$ content of the six mitotypes ranges from $45.19 \%$ in nap to $45.33 \%$ in car, with slight differences among mitotypes. Similarly, nucleotide base content varies slightly in the six mitotypes. The total length of genes with known functions differs between the mitotypes. The percentages that the total length of genes with known functions accounts for the mitochondrial genomes are almost the same in the cam, jun, ole, pol, and nap mitotypes, but the percentage in car is $27.98 \%$ (Table 1), less than that of the other five mitotypes, perhaps as a result of differentiation of Brassica mitotypes.

Analysis of genes with known functions showed that these six mitotypes share 36 species of protein-encoding genes, 3 species of ribosome genes, and 15 species of tRNA genes (Table 2); paralogous genes present in more than one copy are counted here as one species. However, the ole and car mitotypes lack the complexIV-related cox2-2 gene found in the other four mitotypes and the CMS-related genes (homologous to orf 224 and orf222) found in the pol and nap mitotypes [22,23]. The cam and jun mitotypes that have identical gene constitution, also lack the CMS-related genes (orf 224 and orf222). The numbers of genes with known functions are almost the same in all six mitotypes, but the total number of genes varies with mitotype, ranging from 53 in car to 95 in ole (Table 2).

Previous studies have reported physical maps of the cam $[18,24]$ and ole $[17,24]$ mitochondrial genomes. We found that the length of cam sequence is almost the same as that obtained from the physical map (219.7 kb vs. $218 \mathrm{~kb}$ ), but because of a large duplication, the length of the ole mitotype is $360.3 \mathrm{~kb}$, much larger than that previously reported from physical mapping (219 $\mathrm{kb}$ ). A reasonable explanation for the discrepancy may be that the large repeats of ole are difficult to detect by physical mapping.

\section{Repeats}

Large repeats are a cause of the formation of the multipartite structure of the Brassica mitochondrial genome, including one master circle and two smaller subgenomic circles, through reversible homologous recombination [18]. A pair of large $(2,427 \mathrm{bp})$ repeats, called the $R B$ repeats, identified in pol and nap is also found in the cam, jun, and ole mitotypes, but only one copy of RB is found in the car mitotype (Figure 2). Two other pairs of large repeats, $\mathrm{R} 1$ and $\mathrm{R} 2$, are also found in ole; $\mathrm{R} 2$ is an mtDNA fragment $141.8 \mathrm{~kb}$ in size, and R1 carries two exons of the nad5 gene and is $3,605 \mathrm{bp}$ in size. One copy of R1 is found in all other mitotypes except the ole mitotype. Car contains two copies of the 6,580 bp $R$ 
Table 1 Gene contents and total length of six mitotypes

\begin{tabular}{|c|c|c|c|c|c|c|}
\hline Feature & $\operatorname{cam}(\%)$ & jun(\%) & ole(\%) & $\operatorname{car}(\%)$ & pol(\%) & nap (\%) \\
\hline Total gene content & 54 & 54 & 95 & 53 & 56 & 55 \\
\hline Protein-coding genes & 34 & 34 & 56 & 33 & 35 & 35 \\
\hline$r R N A$ & 3 & 3 & 4 & 3 & 3 & 3 \\
\hline $\operatorname{tRNA}$ & 18 & 18 & 35 & 17 & 18 & 17 \\
\hline Total gene length & 67729(30.82) & 67732(30.82) & 112418(31.20) & $64976(27.98)$ & 68408(30.62) & $68415(30.84$ \\
\hline Protein exons & $31620(14.39)$ & 31620(14.39) & $52753(14.64)$ & $30297(13.05)$ & $32295(14.46)$ & $32259(14.54$ \\
\hline Protein introns & $29590(13.47)$ & 29593(13.47) & $48722(13.52)$ & $28234(12.16)$ & 29594(13.25) & $29711(13.39$ \\
\hline$r R N A$ & $5143(2.34)$ & $5143(2.34)$ & $8263(2.29)$ & $5143(2.21)$ & $5143(2.30)$ & $5143(2.32)$ \\
\hline tRNA & $1376(0.63)$ & $1376(0.63)$ & $2680(0.74)$ & $1302(0.56)$ & $1376(0.62)$ & $1302(0.59)$ \\
\hline
\end{tabular}

The percentage of gene length accounting for the entire genome is indicated in parentheses. Cam, jun, ole, and car denote the mitotype of $B$. rapa, $B$. juncea, $B$. oleracea, and B. carinata, respectively. And the pol and nap denote two mitotypes of $B$. napus.

repeats that are not homologous to the repeats of $\mathrm{RB}$, R1 (Figure 2), one copy of RB, and one copy of R1. Therefore, the multipartite structures of cam, jun, pol, and nap might result from the same large RB repeats, and the multipartite structure in car might result from $R$ repeats, but the multipartite structure in ole is complex and is not predicted because it has multiple large repeats. The sizes of multipartite circles for the other five mitotypes except ole predicted by this inference are given in Additional file 1, Table S1.

Tandem repeats exist in all six mitotypes. Frequency distribution analysis of the six mitotypes shows that the size of tandem repeat is mainly between 11 and $40 \mathrm{bp}$, and median of the number of repeats is mainly 2-3 (Additional file 1, Tables S2 and S3). Short repeats range from 30 to $500 \mathrm{bp}$ in length with a total number

Table 2 Gene contents of Brassica mitotypes

\begin{tabular}{|c|c|c|c|c|c|c|c|c|c|c|c|c|c|c|c|}
\hline Product group & Gene & cam & jun & pol & nap & ole & car & Product group & Gene & cam & jun & pol & nap & ole & car \\
\hline \multirow[t]{9}{*}{ Complex I } & nad1 & + & + & + & + & + & + & Ribosome & rps3 & + & + & + & + & +2 & + \\
\hline & nad2 & + & + & + & + & + & + & & rps 4 & + & + & + & + & + & + \\
\hline & nad3 & + & + & + & + & +2 & + & & rps7 & + & + & + & + & +2 & + \\
\hline & nad4 & + & + & + & + & +2 & + & & rps 12 & + & + & + & + & +2 & + \\
\hline & nad4L & + & + & + & + & +2 & + & & rps 14 & + & + & + & + & +2 & + \\
\hline & nad5 & + & + & + & + & +2 & + & & rp/2 & + & + & + & + & +2 & + \\
\hline & nad6 & + & + & + & + & +2 & + & & $r p / 5$ & + & + & + & + & +2 & + \\
\hline & nad7 & + & + & + & + & + & + & & $r p / 16$ & + & + & + & + & +2 & + \\
\hline & nadg & + & + & + & + & +2 & + & tRNA & & & & & & & \\
\hline Complex III & $c o b$ & + & + & + & + & +2 & + & Asparagine & $\operatorname{trnN}$ & + & + & + & + & +2 & + \\
\hline \multirow[t]{4}{*}{ Complex IV } & $\operatorname{cox} 1$ & + & + & + & + & +2 & + & Aspartic & $\operatorname{trn} D$ & + & + & + & + & +2 & + \\
\hline & $\cos 2-1$ & + & + & + & + & +2 & + & Cysteine & $\operatorname{trn} C$ & + & + & + & + & +2 & + \\
\hline & $\cos 2-2$ & + & + & + & + & - & - & Glutamic & $\operatorname{trn} E$ & + & + & + & + & +2 & + \\
\hline & $\cos 3$ & + & + & + & + & + & + & Glutamine & $\operatorname{trn} Q$ & + & + & + & + & +2 & + \\
\hline \multirow[t]{5}{*}{ Complex V } & atp1 & + & + & + & + & +2 & + & & $\operatorname{trn} G$ & + & + & + & + & + & + \\
\hline & atp4 & + & + & + & + & +2 & + & Histidine & $\operatorname{trnH}$ & +2 & +2 & +2 & + & +4 & + \\
\hline & atp6 & + & + & + & + & +2 & + & Isoleucine & trnl & + & + & + & + & +2 & + \\
\hline & atp8 & + & + & + & + & + & + & Lysine & $\operatorname{trnK}$ & + & + & + & + & +2 & + \\
\hline & atp9 & + & + & + & + & + & + & Methionine & $\operatorname{trnM}$ & + & + & + & + & +2 & + \\
\hline \multirow[t]{5}{*}{ Cytochrome c } & $c c m B$ & + & + & + & + & +2 & + & fMethionine & $\operatorname{trnfM}$ & + & + & + & + & +2 & + \\
\hline & $\mathrm{ccmC}$ & + & + & + & + & + & + & Proline & $\operatorname{trn} P$ & + & + & + & + & +2 & + \\
\hline & ccmFN1 & + & + & + & + & + & + & Serine & $\operatorname{trns}$ & +3 & +3 & +3 & +3 & +6 & +3 \\
\hline & ccmFN2 & + & + & + & + & +2 & + & Tryptophan & $\operatorname{trn} W$ & + & + & + & + & +2 & + \\
\hline & ccmFC & + & + & + & + & + & + & Tyrosine & $\operatorname{trn} Y$ & + & + & + & + & +2 & + \\
\hline \multirow[t]{3}{*}{ Other ORF } & tatC & + & + & + & + & +2 & + & $r R N A$ & $r r n 5$ & + & + & + & + & + & + \\
\hline & matR & + & + & + & + & +2 & + & & rrn18 & + & + & + & + & + & + \\
\hline & orf $222 / 4$ & - & - & + & + & - & - & & $r r n 26$ & + & + & + & + & +2 & + \\
\hline
\end{tabular}




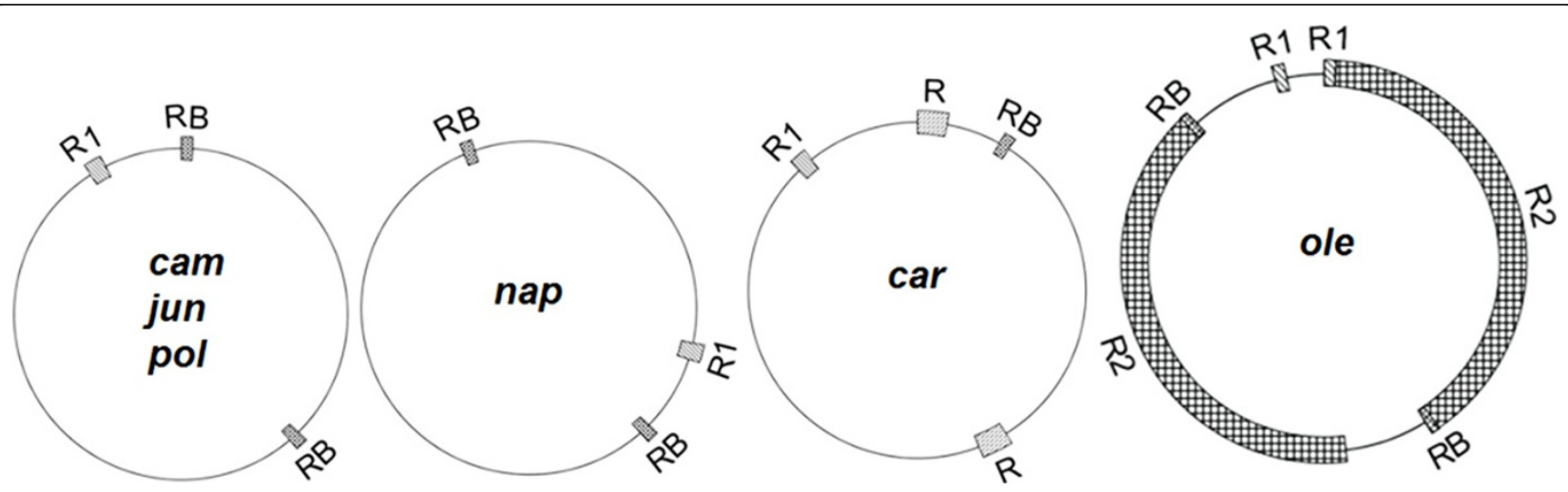

Figure 2 Large repeats exist in the six mitotypes. RB, R1, R, and R2 denote repeats of more than $2 \mathrm{~kb}$. RB and R1 are shared by the six mitotypes, but their copy numbers vary.

of approximately 190 , accounting for roughly $5 \%$ of the entire mitochondrial genome.

Short repeats are closely related with an irreversible re-organization of the Brassica mitochondrial genomes $[7,25,26]$. The short repeats are uniformly distributed in the six mitochondrial genomes, as detected by the Kolmogorov-Smirnov method [27]. This may imply that there are prerequisites for the irreversible rearrangement. To investigate the relationships of syntenic region rearrangements, syntenic regions were numbered (defined in Figure 3). We found that some rearrangements of numbered regions are associated with short repeats. For example, Figure 4A shows that in the cam and ole mitotypes, syntenic segments $7,2,5,6,8$, and 1 were rearranged together with sequence direction changes, and the recombinations that resulted in these rearrangements are related to the short repeats marked $\mathrm{Q}$, which are made up of approximately $100 \mathrm{bp}$ repeats within the neighboring segment. Another typical example is the short repeat of about $70 \mathrm{bp}(\mathrm{P})$ : the separated segments 16 and 1 that contain the short $\mathrm{P}$ repeat in the car mitotype were recombined into the rearranged form in ole omitting the $\mathrm{P}$ repeat (Figure 4B). These findings are evidence for an association of short repeats with rearrangements.

A $141.8 \mathrm{~kb}$ segment duplication in the ole mitotype was the main factor influencing the differences in the number of functional genes between ole and the other five mitotypes. This large duplicated 38 genes with known functions and several partial exons, including three exons of nad1, three exons of nad2, and three exons of nad5. This partial duplication of the mitochondrial genome and the resulting dramatic change in the copy number of genes and ORFs of B. oleracea suggests that the role of ole mitotype is different from that of the other five Brassica mitotypes in cytotypenuclear genotype interactions or plant-environment interactions.
Inheritance of mitochondrial genomes in Brassica

By comparing the complete sequences, we found that the cam and jun mitotypes have the same genome organization and are closely related at the nucleotide sequence level. According to U's triangle for the cytological relationship between Brassica species, B. juncea is derived from the offspring of an interspecific cross between $B$. rapa and B. nigra; thus, the cam mitotype of $B$. rapa can be inferred to have been transmitted into $B$. juncea without a significant genomic change. Similarly, the pol mitotype in B. napus, which is postulated to be derived from the offspring of an interspecific cross between B. rapa and B. oleracea according to U's triangle, has only one region different from cam, suggesting that $\mathrm{pol}$ also inherited the cam mitotype, with an evolutionary modification. At the modification position, pol has a 4.4-kb insertion and cam has an 813-bp insertion, suggesting that pol was derived from cam at this location through evolutionary modifications resulting in a 813-bp sequence deletion and 4.4-kb sequence insertion. The relationship between pol, jun, and cam shows that the cytoplasmic origin of plant species can be traced because the mitochondrial genome is conserved. On the other hand, this pattern of mitotype inheritance in plants, for which we are presenting the first evidence, indicates that the evolution of new composite species is not always accompanied by sudden mitochondrial genome changes, or that new species may inherit maternal mitochondrial genomes with a few insertions/deletions (indels), as shown in the pol mitotype. This mitotype inheritance mechanism might be involved in other composite plant species formed by chromosomal doubling or interspecies hybridization.

\section{Mitochondrial genome restructuring}

Relative to three highly similar mitotypes (cam, jun, and pol), nap, ole, and car were found to be restructured mitotypes. The genomic structure of the ole mitotype is 


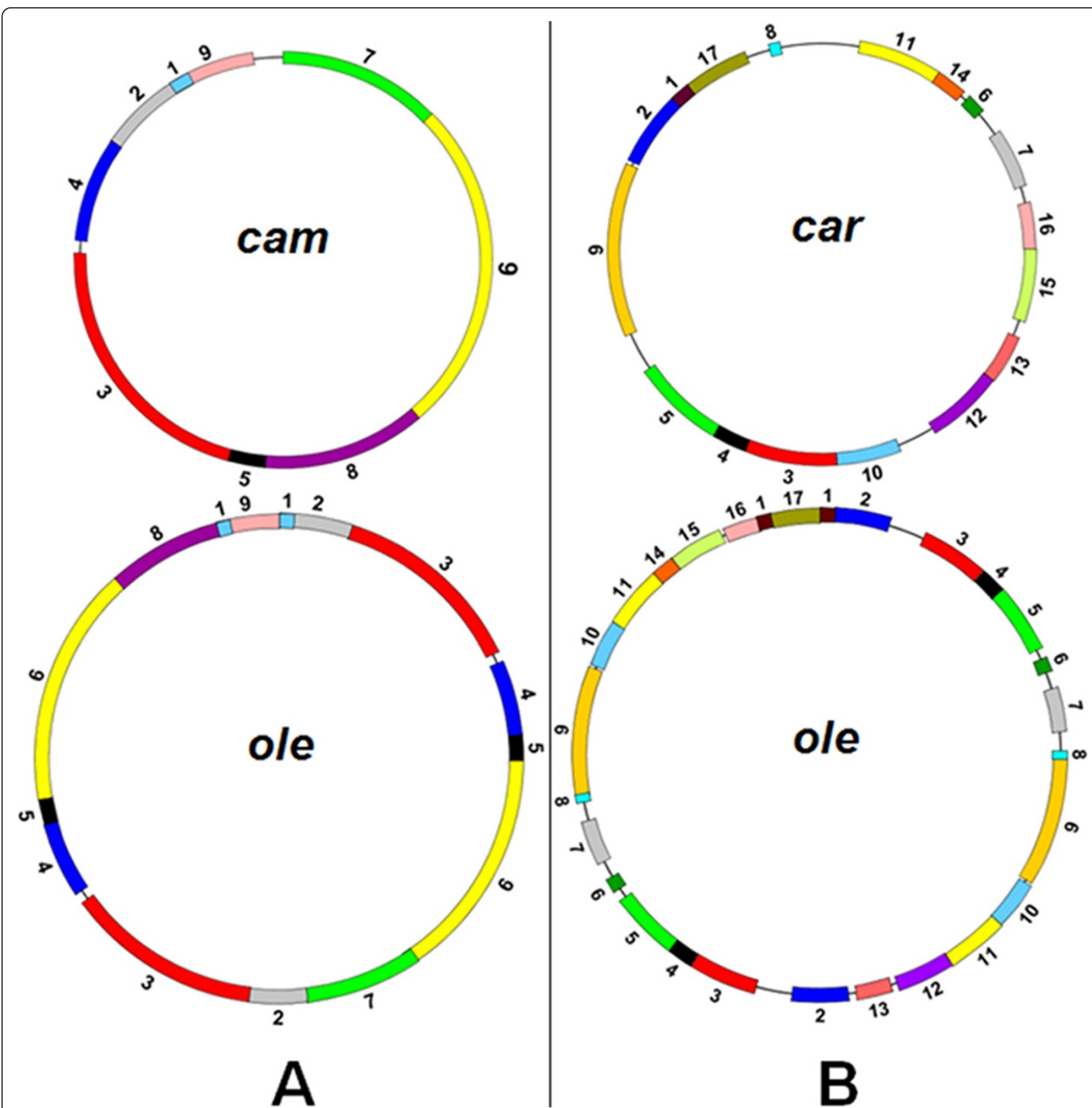

Figure 3 Rearrangements of Brassica mitochondrial genomes. Syntenic regions $>2 \mathrm{~kb}$ are shown. (A) Rearrangement of the cam mitochondrial genome with the ole mitotype as a reference. (B) Rearrangement of the car mitochondrial genome with the ole mitotype as a reference. The numbers refer to the syntenic regions derived from a paired comparison. Highly or completely homologous regions are indicated by color.

different from that of cam (Figure 3A), and it can be inferred that, relative to cam, the genomic structure of ole has experienced at least four recombination events. The recombination events and a large duplication (141.8 $\mathrm{kb}$ ) (Figure 2) made the ole mitotype distinct from the other five mitotypes. Relative to ole, the car mitochondrial genome has as many as 17 syntenic regions (Figure $3 \mathrm{~B})$, suggesting that the car mitotype restructuring is more complex. The number of recombination events in the process of mitochondrial genome formation in car is difficult to estimate, because there are so many syntenic regions.

The similarities between the syntenic regions (Additional file 2) of the six Brassica mitotypes are very high, with the lowest nucleotide identity being $87 \%$ and most of the nucleotide identity being more than 99\%, 


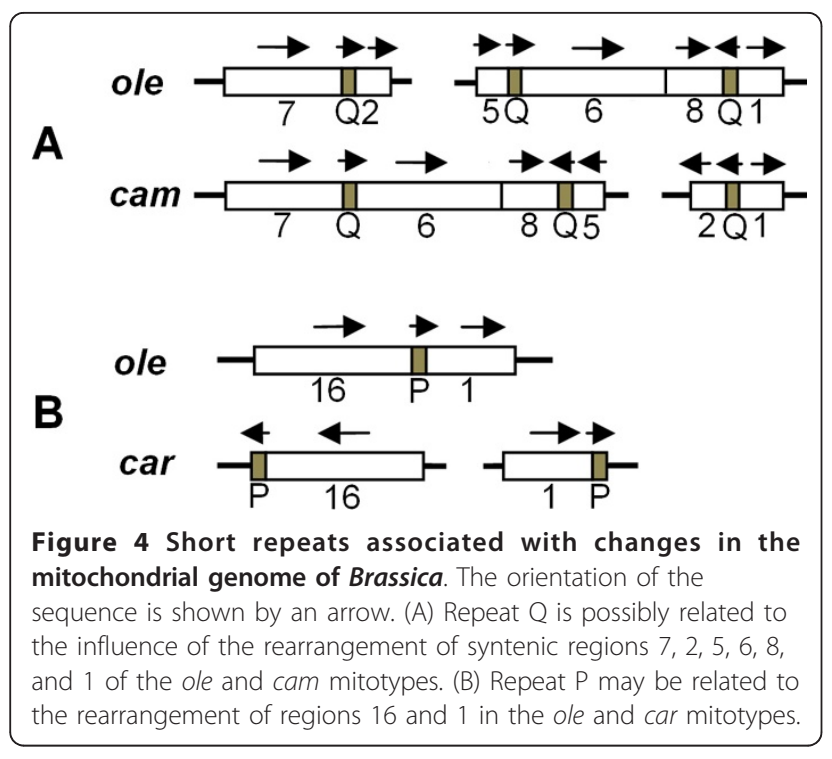

indicating that the syntenic regions are consistently conserved in Brassica.

\section{Mitotype divergence}

Structural comparisons between two mitotypes help to identify indel sequences present in one mitochondrial genome and absent (or deleted) in another. Indels frequently found in both coding and non-coding gene regions of plant mitochondrial genomes can provide evolutionary clues $[8,28]$. To illustrate the genomic differences, we summed the length of indels found in a mitochondrial genome comparison and defined a dissimilarity indicator to measure the divergence between two mitochondrial genomes using indels and single nucleotide polymorphisms (SNPs) between them. Cluster analysis of the six mitotypes showed that cam and pol are in one class, ole slightly diverges from the campol class, and nap and car have diverged the furthest from the cam-pol group (see Figure 5 and Additional file 1, Tables S4 and S5).

Combined indel analysis of multiple mitochondrial genomes showed that all insertions in the cam, jun, pol, and ole mitotypes can be aligned with other mitotypes. However, for some insertions in the nap and car mitotypes, matching sequences with high homology could not be found in other Brassica mitotypes. These unmatched insertions account for $2.92 \%$ of the nap mitotype (one insertion larger than $2 \mathrm{~kb}$ ) and $10.36 \%$ of the car mitotype (11 insertions).

Variation of open reading frames with unknown functions Using the ORF Finder software at NCBI, the cam, pol, nap, ole, and car mitotypes were found to contain 44, $45,46,44$, and 36 species of ORFs with unknown functions, respectively, indicating that the numbers of ORFs in the six mitotypes are different (jun has an ORF constitution identical to cam). On the other hand, the six mitotypes share 20 ORFs with unknown functions: orf100b, orf101a, orf101b, orf104, orf106b, orf108b, orf109, orf113a, orf115a, orf115c, orf115d, orf116, orf119, orf120, orf135, orf146, orf159, orf161, orf257, and orf448. Another 34 ORFs sequences are not possessed consistently by all of the six Brassica mitotypes (Table 3) (defined here as polymorphic ORFs, present in one mitotype in complete form, and completely absent or present in partial or mutated form in other mitotypes).




Table 3 Function-unknown ORFs predicted by ORF Finder in six Brassica mitotypes

\begin{tabular}{|c|c|c|c|c|c|c|c|c|c|c|c|c|c|}
\hline ORF & cam & pol & nap & ole & car & class & ORF & cam & pol & nap & ole & car & class \\
\hline orf101c & + & + & + & $2+$ & - & $G R$ & orf106a & - & - & + & $2+$ & + & $M$ \\
\hline orf265 & + & + & orf261 & $\operatorname{orf} 266^{*}(2+)$ & orf277 & $G R$ & orf108a & + & + & + & + & - & M \\
\hline orf305 & + & + & orf322* & $\operatorname{orf311}(2+)$ & $\operatorname{orf310^{*}}$ & GR & orf110 & + & + & + & $2+$ & - & M \\
\hline orf101d & + & + & + & $2+$ & - & ID & orf112 & + & + & + & $2+$ & - & M \\
\hline orf117b & - & - & + & $2+$ & + & ID & orf113b & + & + & + & $2+$ & orf170 & M \\
\hline orf122 & + & + & - & $2+$ & - & ID & orf114 & + & + & + & - & + & M \\
\hline orf124 & - & - & - & - & + & ID & orf115b & + & + & + & $2+$ & - & M \\
\hline orf128 & + & + & + & $2+$ & - & ID & orf117a & + & + & + & + & - & M \\
\hline orf132 & + & + & - & $2+$ & - & ID & orf118 & + & + & orf124 & orf120a & orf123a & M \\
\hline orf138a & - & - & - & - & + & ID & orf123 & + & + & + & $2+$ & - & M \\
\hline orf188 & - & - & + & - & - & ID & orf125 & + & + & + & $2+$ & orf108c & $M$ \\
\hline orf195 & + & + & + & - & - & ID & orf128a & - & - & - & - & + & $M$ \\
\hline orf $222^{\#}$ & - & orf224 & orf222 & - & - & ID & orf131 & - & - & - & - & + & $M$ \\
\hline orfiooa & + & + & + & $2+$ & orf100c & M & orf147 & + & + & + & + & orf141 & $M$ \\
\hline orfiole & + & + & + & $2+$ & - & M & orf164 & + & + & + & + & - & $M$ \\
\hline orf101f & + & + & + & + & - & $M$ & orf165 & - & - & - & - & + & $M$ \\
\hline orf105 & - & - & - & $2+$ & - & M & orf293 & + & + & orf286 & - & orf288 & $M$ \\
\hline Total nu & ber & Incti & , & in & & & & $24(44)$ & $25(45)$ & $26(46)$ & $24(44)$ & $16(36)$ & \\
\hline
\end{tabular}

Each line presents the homologous ORFs. +, present; -, absent; GR, gene rearrangement; ID, insertion/deletion; M, mutation. ORF copy numbers are shown ahead of + . Class shows the cause of change in each line.

* indicates an exception for class and that the cause is mutation.

\# orf222 and orf224 are the same as Table 1.

Data for pol and nap are from our previous report and Handa's results [6], respectively. The total numbers outside parentheses show the changing ORF for each mitotype; the number in parentheses contains the ORFs that exist consistently in the six mitotypes. The total number of ORFs in ole is not plus the multiple copies, if containing the copies the total number is 73 .

This result suggests that the inclusion of ORFs with unknown functions in different mitotypes are very different.

Genome rearrangement may result in loss of ORFs through a DNA break within an ORF, which results in loss of the ORF or generation of a novel ORF through the recombination of the broken sequences with others. In our analysis, three polymorphic ORFs were found to be due to genome rearrangement (Table 3). For example, the breakage of orf101c at position +108 due to genome rearrangement has led to the loss of this ORF in car, and the orf265 sequence in nap was lost in the course of rearrangement, generating orf261 [7]. In car, breakage of the orf 265 sequence at position +463 was followed by recombination of its terminal sequence with another sequence, resulting in orf 277 of the car mitotype.

Ten polymorphic ORFs were caused by indels (Table 3 ), indicating that indels had a direct influence on ORF variation. Orf101d and orf128 are absent in car, orf $117 b$ is absent in cam and pol, orf 122 and orf 132 are absent in nap and car, and orf124 and orf138a exist only in car. The region including orf 224 in pol and $\operatorname{orf} 188$, orf 222 in nap is contained in an insert region, as shown in comparison of the two mitotypes (Additional file 2).
Twenty-one out of the 34 polymorphic ORFs resulted from nucleotide mutations (Table 3), suggesting that mutations have a more important role than other evolutionary events in mitotype formation in Brassica. There are a total of 105 mutation sites in the 21 polymorphic ORF sequences, of five mitotypes, of which 67 sites are frame-preserving mutations although the length of ORF is changed, three sites are single nucleotide mutation sites resulting in premature stop codons, four are triplet indels of less than 52 nucleotides in length (in orf $120 a$, orf108c, orf286, and orf288), which cause length variation among the homologous ORFs, and the other 31 sites are non-triplet indels, including two sites of $1 \mathrm{bp}$ indels, six sites of 2 bp indels, four sites of 4 bp indels, five sites of $5 \mathrm{bp}$ indels, and 14 sites of indels more than 5 bp. For example, orf118 is incomplete because of several nucleotide mutations in car. In the ole mitotype, mutation of nucleotides TC to AA at positions +188 and +189 in orf120a occurred together with a AGTATT insertion at +212 to generate orf120a.

Little is known about the roles of the predicted ORFs, because limited studies have been carried out on them. Only one ORF, the CMS-related orf 222 (or its homolog orf224), has been demonstrated to be important, but the ORFs with unknown functions specific to Brassica could 
have a particular role in the mitochondrial activities of a particular species [29].

Given the above-mentioned numbers of polymorphic ORFs with unknown functions, the importance of the roles of the three evolutionary factors in genome formation may be arranged in the following order: mutation > segment indel $>$ genome rearrangement. The mechanism including these evolutionary factors undoubtedly changed the mitochondrial genome structure and ORFs, but these factors influencing the Brassica mitochondrial genomes did not alter the constitution of the gene species (or classes) with known functions, which is almost same as the situation in A. thaliana [1]. This result suggests that genes with known functions in the mitochondrial genomes are not changed, and it may indicate that the genes with known functions are too important to be lost or indispensable across the long evolutionary time in the plant genus or within several closely related species.

\section{Discussion}

\section{Mitotype formation in Brassica}

In previous analyses of plant mitochondrial genomes, several evolutionary factors, such as duplication, rearrangement, indel, and mutation, have been found to be important in genome evolution $[5,8,9,30]$. In this study, as well as these evolutionary factors, we have shown for the first time that mitotype inheritance can explain well the formation of some of the mitotypes ( $\mathrm{pol}$ and jun) of Brassica. Thus, we suggest that the Brassica mitotypes are derived through a mechanism including several evolutionary events, such as inheritance, duplication, rearrangement, indel, and mutation of the plant mitochondrial genome sequence. However, this is not our final conclusion on mitochondrial genome formation because this cannot explain the phenomena resulting from our syntenic region alignment.

We have inferred the origin of two mitotypes ( $\mathrm{pol}$ and jun), but how have the other four mitotypes originated? We analyze this result as follows.

First, U's triangle can help reveal the origin of mitotypes (Figure 1). From the cytogenetics of Brassica, the composite species have been thought to be formed by interspecific hybridization between elementary species and following chromosome doubling, and the mitotypes of composite Brassica may be regarded as having been derived by inheritance from corresponding maternal parents with primary mitotypes. In the past, the maternal parents of amphiploid species were inferred to be the species with more chromosomes on basis of the crossability between two parents. For example, B. rapa is regarded as the ancestral female parent for $B$. napus because a cross with $B$. rapa as maternal parent and $B$. oleracea as male parent is easier to accomplish than its reverse cross, and mitotypes of $B$. napus including nap can be regarded as inherited from the cam mitotype ( $B$. rapa) although this lacked evidence at the molecular level.

Second, our combined insertion alignment results show that the insertions in the cam, jun, pol and ole mitotypes can be aligned with other Brassica mitotypes. This result implies that, if we regard cruciferous species with bigger mitochondrial genomes than most Brassica as ancestral species, the Brassica mitotypes can be inferred to have evolved from a common ancestral parent with all the mitochondrial genome information of Brassica mitotypes, and which existed after the so-called mitochondrial genome expansion of angiosperms [31]. The indels in Brassica mitotypes may be traces of deletions from the common ancestral parent. If we do not accept this hypothesis, then we must postulate that the insertion sequences were obtained through horizontal transfer or transfer from the nuclear genome [32,33]. The possibility that insertion sequences are transfers from the nuclear genome can be ruled out because none of them can be aligned with the nuclear DNA in a search of NCBI sequence databases. As for insertion sequences from horizontal transfer between mitochondria, the concept is seemingly logical but currently lacks supporting evidence. In addition, the so-called transfers have been considered to be random transfers, so we cannot conceive why the same insert sequences are found in different mitotypes. Thus, we conclude that there has been some genome compaction during the process of mitochondrial genome evolution.

Third, there are two mitotypes identified in B. napus, the nap and pol mitotypes [7,18]. The nap mitotype, discovered by Shiga and Thompson [34,35], exists popularly in natural rapeseed germplasm population with usually male fertile phenotype in most genetic backgrounds. The pol mitotype, which is sparse in natural rapeseed germplasm, resulting in cytoplasmic male sterility with easily-found restorer lines, was discovered first by $\mathrm{Fu}$ [36], has been widely used in to generate heterosis. No other natural mitotype apart from these two mitotypes of B. napus has been identified. Also, no other natural mitotype for the elementary species of Brassica apart from those studied here has been identified, and thus how the nap mitotype has been inherited appears to remain unsolved. Nevertheless, the nap mitotype might have been inherited from an unidentified or lost mitotype of B. rapa, which has very rich germplasm, and then experienced mitochondrial genome evolution events as mentioned above. Similarly, the formation of the car mitotype found in the composite species B. carinata can be explained by the same mechanism as that proposed for the nap mitotype. Our previous results [7] demonstrated that the nap and pol mitotypes coexist in 
B. napus: the pol cytoplasm consists mainly of the pol mitotype, and the nap mitotype is the main genome of nap cytoplasm. In this case, the inference that the maternal parent of nap mitotype is $B$. rapa, is further supported because the coexistence does not contradict the genome compaction hypothesis. Mitotype coexistence has also been demonstrated in other plant species, such as Phaseolus vulgaris $[37,38]$. Whether mitotype coexistence can be regarded as vestige of ancestral maternal mitochondrial genome compaction needs to be further explored, but the compaction may be a cause of mitotype diversification for the different species with different nuclei (speciation) as a result of adaptation of each plant species to internal and external environment.

Finally, from the above analysis, mitotypes for elementary species of Brassica can be regarded as the products of condensation or compaction of a bigger ancestral mitochondrial genome accompanied by long-term evolution history changes that may be necessary for adaptation to their environments, or be random evolutionary events such as mutation. Composite species may be inferred to have been derived from maternal mitotype inheritance and the following modification of maternal mitotype (Figure 6).

Additionally, intergenic regions of the mitochondrial genome were not found to be conserved in other studies $[30,39]$, but our results on intergenic regions seem to be inconsistent with this conclusion. A reasonable explanation is that the mitotypes in our analysis belonging to the same genus in which closely related species were formed through genome compaction. In previous studies, either the analyzed materials were not as closely related as in our study, or the origins of the indels (the so-called non-conserved regions in other studies) were not elucidated. Our results may be an addition to the evolutionary theory of the mitochondrial genome.

\section{Evolutionary pattern of plant mitochondrial genomes}

Mitochondrial genomes are considered to be have evolved monophyletically from a eubacterial ancestor $[31,40]$. The evolutionary trends are different between animals and plants over their long evolutionary history. The trend in animals is towards a further compaction of the mitochondrial genome through loss of genes and intergenic spacers, as exemplified in Homo sapiens and Metridium senile [41-43]. Conversely, the mitochondrial genomes in plants and fungi have experienced mtDNA expansions, including increases in size, primarily by acquisition of a large amount of apparently non-coding DNA of currently unknown origin and function $[41,43]$. This description is summarized as the serial endosymbiosis theory, which remains unrefuted $[31,41]$.

Nevertheless, our research with Brassica as a phylogenetic model clade has demonstrated that a compaction mechanism has been involved in plant mitotype evolution. This phylogenetic pattern within a Brassica clade

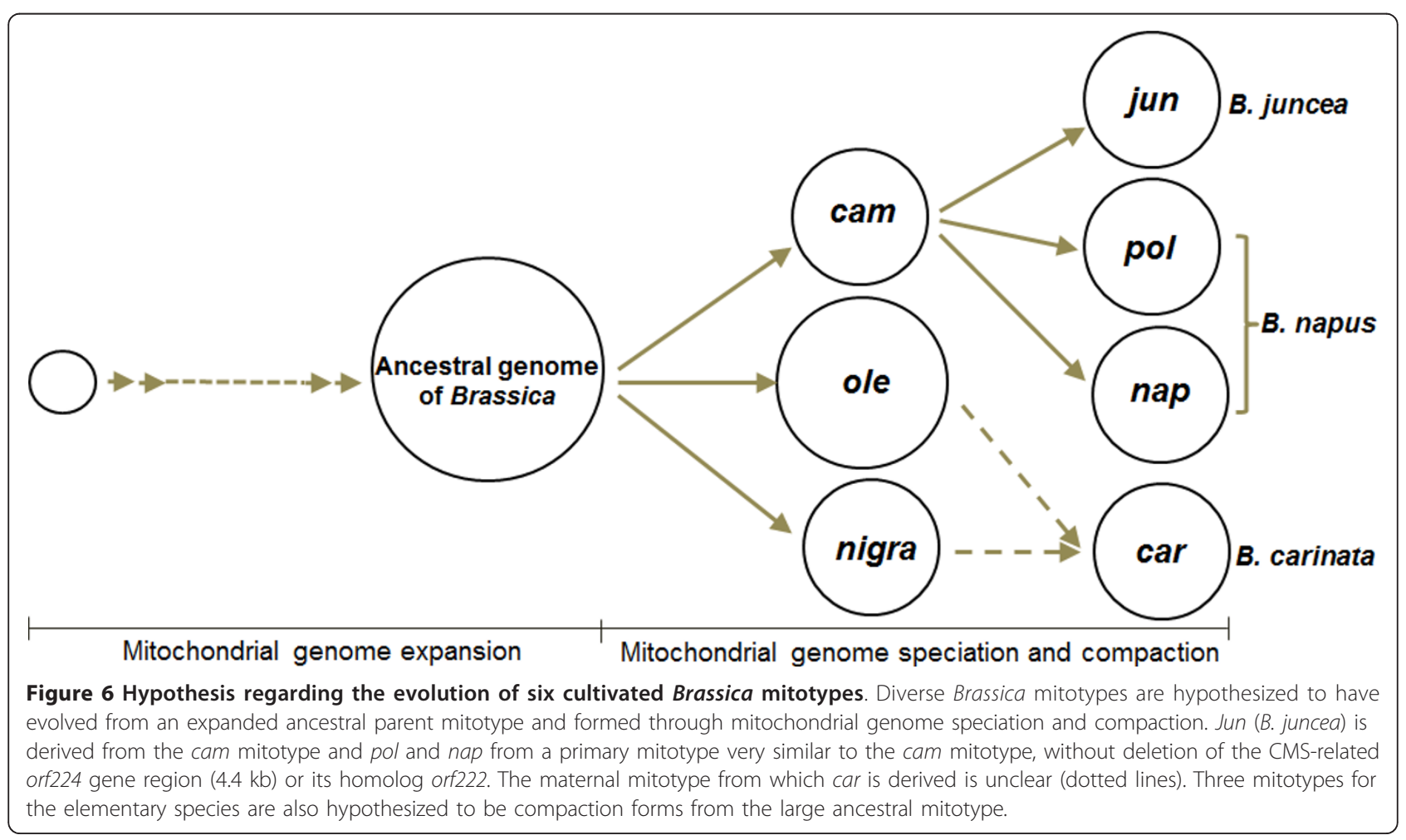


coincides well with the properties of the animal clade. Given this conclusion, one difference in the evolutionary mechanism between animals and angiosperms may be that the mitochondrial genome has undergone an expansion process in angiosperms but not in animals.

\section{Mitotype effects}

Rapeseed breeders pay much attention to cytoplasmic effects. From our results, the jun and cam mitotypes do not have the CMS-related orf222 gene found in nap or the homologous CMS-related orf 224 found in pol, and other genes with known functions are almost the same as in the nap, pol or jun mitotype, so jun and cam may probably be beneficial to breeding of conventional varieties of $B$. napus because the CMS-related gene is usually regarded as being noxious to plants [44]. The fact that the amphiploid B. juncea is more tolerant to stressful environments than $B$. napus [21] is probably related to the mitotype differences between $B$. juncea and $B$. napus. Potential uses of the ole and car mitotypes in Brassica crop breeding need to be explored.

\section{Conclusions}

This study has compared the sequences of six Brassica mitotypes. The pol mitotype of $B$. napus and the jun mitotype of $B$. juncea are found to be an inherited form of the cam mitotype with less modification. This result may have implications for the mitochondrial genome origin of other composite plant species formed by chromosomal doubling or interspecific hybridization. Sequence relationship analyses showed that a mechanism of genome compaction and inheritance has been involved in Brassica mitotype evolution. Our study suggests a mechanism of mitochondrial genome formation in Brassica including evolutionary events such as inheritance, duplication, rearrangement, sequence compaction (indels), and mutation.

\section{Methods}

\section{Mitochondrial DNA preparation}

Materials used for mitochondrial genome sequencing included four Brassica accessions: Suzhouqing of $B$. rapa, Jiangpu-yejiecai of $B$. juncea, $08 C 717$ of $B$. oleracea, and W29 of B. carinata. The first two of these were from our germplasm deposited in the National Key Lab of Crop Genetics and Germplasm Enhancement in Nanjing Agricultural University. The 08C717 accession of B. oleracea was provided by the Institute of Vegetable Crops in Jiangsu Academy of Agricultural Sciences, and W29 of B. carinata was provided by Yanyou Wu of Jiangsu University. Methods for mitochondrial DNA extraction and purification of the four species were reported previously [7].

\section{Genome sequencing}

The Brassica mtDNA was sequenced using the massively parallel GS-FLX DNA pyrosquencing platform from Roche 454 Life Sciences (Branford, CT, USA). Contigs were joined by Sanger sequencing of PCR products. The number of reads for cam, jun, ole, and car were $11,805,11,125,15,189$, and 29,676, respectively. Total sequence data for cam, jun, ole, and car were 4,432,258 bp, 4,422,401 bp, 6,798,916 bp, and 13,381,537 $\mathrm{bp}$, respectively, representing mitochondrial genome coverage ratios of 19X, 20X, 18X, and 57X, respectively. The coverage ratio for the car mitotype is far greater than that of the others because it was sequenced twice, as the first sequencing attempt was not successful.

\section{Genome annotation}

Database searches were carried out using the NCBI web-based Blast service [45], and genes with e-values less than 0.001 were selected. The ORFs were used to query non-redundant databases using BLAST similarity searches, by applying a cut-off of $70 \%$ sequence identity over at least $80 \%$ of the ORF length. To define genes, ORF Finder, BLASTN, BLASTX [45], and tRNAscan-SE [46] were used. To improve identification accuracy, the mtDNA annotations were compared with other plant mitochondrial genome annotations, and all differences in coding predictions were reassessed on the basis of the choice of the start codon, length of plant mtDNA conservation, and the presence of identical motifs. In addition to unknown genes, only ORFs of at least 100 codons were annotated, using the mitochondrial genome annotations of the nap (GenBank: AP006444.1) and pol (EMBL: FR715249) mitotypes of B. napus as a reference.

\section{Mitochondrial genome comparisons}

The six mitochondrial genomes were aligned using BLASTN [45]. Homologous segments > 400 bp in length were chosen. Indel, defined as a sequence present in one mitochondrial genome but absent in another mitochondrial genome, were extracted to elucidate genomic divergence. Two previously reported mitochondrial genomes for Brassica were combined for analysis. The dot matrix plot provided in BLASTN helped in analysis of genome restructuring. Additionally, progressive Mauve [47] was used to identify SNPs between genomes.

\section{Analysis of repeats}

Repeats (30-500 bp) were discovered using in-house private commercial software developed by Shanghai Majorbio Bio-pharm Biotechnology Company (China). BLASTN was used to identify repeats longer than 500 bp. Information on tandem repeats was obtained using the tandem repeats finder [48]. The Kolmogorov- 
Smirnov method was used to test the uniformity of short repeat distribution.

\section{Mitochondrial genome clustering}

Two main indicators were used to measure mitochondrial divergence: the number of SNPs between two genomes and the total length of indel sequences that account for differences when two mitochondrial genomes were compared. Dissimilarities among mitochondrial genome sequences were measured with the formula $d=\left(2 N_{S N P}+L_{\text {Indel } 1}+L_{\text {Indel } 2}\right) /\left(L_{G 1}+L_{G 2}\right)$, where $L_{G 1}$ and $L_{G 2}$ represent the entire lengths of the two mitochondrial genomes to be compared, $N_{S N P}$ represents the number of SNPs found when the paired genomes were compared, and $L_{\text {Indel } 1}$ and $L_{\text {Indel } 2}$ denote the total length of insertion sequences for the two mitochondrial genomes. With this definition of dissimilarity, the UPGMA method [49] was used to cluster the mitochondrial genomes of Brassica species.

\section{Additional material}

Additional file 1: Supplemental Tables S1 to S5 providing detailed analyses results

Additional file 2: The syntenic regions derived from mitotype-tomitotype comparisons

\section{List of abbreviations}

bp: base pairs; CMS: cytoplasmic male sterility; indel: insertion/deletion; mtDNA: mitochondrial DNA; SNP: single nucleotide polymorphism; ORFs: open reading frames.

\section{Acknowledgements}

This work was supported by the National Basic Research Program of China (973 Program) (2011CB109300), National Natural Science Foundation of China (30970289) and National Key Technology R\&D Program

(2011BAD13B09) in China. The authors wish to thank Prof. Hongsheng Zhang from Nanjing Agricultural University (China) for his help to our experiment, and thank Shanghai Majorbio Bio-pharm Biotechnology Company (China) for the sequencing of the mtDNA.

\section{Author details}

${ }^{1}$ State Key Laboratory of Crop Genetics and Germplasm Enhancement, Nanjing Agricultural University, Nanjing 210095, PR China. ${ }^{2}$ Institute of Vegetable Crops, Jiangsu Academy of Agricultural Sciences, Nanjing 210014, PR China.

\section{Authors' contributions}

SC carried out the experiments and performed sequence analysis. TY, YH, JC and $J Y$ participated in the experiments. TD, JH participated in the sequence analysis. RG conceived and supervised the work, guided sequence analysis and drafted the manuscript. All authors read and approved the final manuscript.

Received: 24 June 2011 Accepted: 11 October 2011

Published: 11 October 2011

\section{References}

1. Unseld M, Marienfeld JR, Brandt P, Brennicke A: The mitochondrial genome of Arabidopsis thaliana contains 57 genes in 366,924 nucleotides. Nature genetics 1997, 15(1):57-61.
2. Kubo T, Nishizawa S, Sugawara A, Itchoda N, Estiati A, Mikami T: The complete nucleotide sequence of the mitochondrial genome of sugar beet (Beta vulgaris L.) reveals a novel gene for tRNA ${ }^{\text {Cys }}(\mathrm{GCA})$. Nucleic Acids Res 2000, 28(13):2571-2576.

3. Notsu Y, Masood S, Nishikawa T, Kubo N, Akiduki G, Nakazono M, Hirai A, Kadowaki K: The complete sequence of the rice (Oryza sativa L.) mitochondrial genome: frequent DNA sequence acquisition and loss during the evolution of flowering plants. Mol Genet Genomics 2002, 268(4):434-445.

4. Fujii S, Kazama T, Yamada M, Toriyama K: Discovery of global genomic reorganization based on comparison of two newly sequenced rice mitochondrial genomes with cytoplasmic male sterility-related genes. BMC Genomics 2010, 11:209.

5. Tian $X$, Zheng J, Hu S, Yu J: The rice mitochondrial genomes and their variations. Plant Physiol 2006, 140(2):401-410.

6. Handa $\mathrm{H}$ : The complete nucleotide sequence and RNA editing content of the mitochondrial genome of rapeseed (Brassica napus L.): comparative analysis of the mitochondrial genomes of rapeseed and Arabidopsis thaliana. Nucleic Acids Res 2003, 31(20):5907-5916.

7. Chen J, Guan R, Chang S, Du T, Zhang H, Xing H: Substoichiometrically different mitotypes coexist in mitochondrial genomes of Brassica napus L. PLoS One 2011, 6(3):e17662.

8. Allen JO, Fauron CM, Minx P, Roark L, Oddiraju S, Lin GN, Meyer L, Sun H, Kim K, Wang C, Du F, Xu D, Gibson M, Cifrese J, Clifton SW, Newton KJ: Comparisons among two fertile and three male-sterile mitochondrial genomes of maize. Genetics 2007, 177:1173-1192.

9. Darraca A, Varre JS, Touzet P: A scenario of mitochondrial genome evolution in maize based on rearrangement events. BMC Genomics 2010, 11:233.

10. Clifton SW, Minx P, Fauron CM, Gibson M, Allen JO, Sun H, Thompson M, Barbazuk WB, Kanuganti S, Tayloe C, et al: Sequence and comparative analysis of the maize NB mitochondrial genome. Plant Physiol 2004, 136(3):3486-3503.

11. Sugiyama $Y$, Watase $Y$, Nagase $M$, Makita $N$, Yagura $S$, Hirai $A$, Sugiura $M$ : The complete nucleotide sequence and multipartite organization of the tobacco mitochondrial genome: comparative analysis of mitochondrial genomes in higher plants. Mol Genet Genomics 2005, 272(6):603-615.

12. Ogihara $Y$, Yamazaki $Y$, Murai $K$, Kanno A, Terachi $T$, Shiina T, Miyashita N, Nasuda S, Nakamura C, Mori N, et al: Structural dynamics of cereal mitochondrial genomes as revealed by complete nucleotide sequencing of the wheat mitochondrial genome. Nucleic Acids Res 2005, 33(19):6235-6250.

13. Goremykin W, Salamini F, Velasco R, Viola R: Mitochondrial DNA of Vitis vinifera and the issue of rampant horizontal gene transfer. Mol Biol Evol 2009, 26(1):99-110.

14. Alverson AJ, Wei XX, Rice DW, Stern DB, Barry K, Palmer JD: Insights into the evolution of mitochondrial genome size from complete sequences of Citrullus lanatus and Cucurbita pepo (Cucurbitaceae). Mol Biol Evol 2010, 27(6):1436-1448.

15. Alverson AJ, Zhuo S, Rice DW, Sloan DB, Palmer JD: The mitochondrial genome of the legume Vigna radiata and the analysis of recombination across short mitochondrial repeats. PLoS One 2011, 6(1):e16404.

16. Palmer JD, Shields CR: Tripartite structure of the Brassica campestris mitochondrial genome. Nature 1984, 307(5950):437-440.

17. Chétritl P, Mathieu C, Muller JP, Vedel F: Physical and gene mapping of cauliflower (Brassica oleracea) mitochondrial DNA. Current Genetics 1984, 8(6):413-421.

18. Palmer J, Herbon L: Plant mitochondrial DNA evolves rapidly in structure, but slowly in sequence. J Mol Biol 1988, 28:87-97.

19. Schuster W, Brennicke A: The Plant Mitochondrial Genome - Physical Structure, Information-Content, RNA Editing, and Gene Migration to the Nucleus. Annu Rev Plant Physiol Plant Molec Biol 1994, 45:61-78.

20. UN: Genome-analysis in Brassica with special reference to the experimental formation of $B$. napus and peculiar mode of fertilization. Japan J Bot 1935, 7:389-452.

21. Gómez-Campo C: Biology of Brassica Coenospecies. Amsterdam: Elsevier; 1999.

22. Singh M, Brown GG: Suppression of cytoplasmic male sterility by nuclear genes alters expression of a novel mitochondrial gene region. Plant Cell 1991, 3(12):1349-1362. 
23. L'Homme Y, Stahl RJ, Li XQ, Hameed A, Brown GG: Brassica nap cytoplasmic male sterility is associated with expression of a mtDNA region containing a chimeric gene similar to the pol CMS-associated orf224 gene. Current Genetics 1997, 31(4):325-335.

24. Manchekar M, Scissum-Gunn KD, Hammett LA, Backert S, Nielsen BL: Mitochondrial DNA recombination in Brassica campestris. Plant Science 2009, 177(6):629-635.

25. Shedge V, Arrieta-Montiel M, Christensen AC, Mackenzie SA: Plant mitochondrial recombination surveillance requires unusual RecA and MutS homologs. Plant Cell 2007, 19(4):1251-1264.

26. Andre C, Levy A, Walbot V: Small repeated sequences and the structure of plant mitochondrial genomes. Trends in genetics 1992, 8(4):128-132.

27. Lopes RHC, Reid I, Hobson PR: The two-dimensional Kolmogorov-Smirnov test. In XI International Workshop on Advanced Computing and Analysis Techniques in Physics Research 2007.

28. Gregory TR: Insertion-deletion biases and the evolution of genome size. Gene 2004, 324:15-34.

29. Kubo N, Arimura S: Discovery of the rp/10 gene in diverse plant mitochondrial genomes and its probable replacement by the nuclear gene for chloroplast RPL10 in two lineages of angiosperms. DNA research 2010, 17(1):1-9.

30. Kubo T, Newton KJ: Angiosperm mitochondrial genomes and mutations. Mitochondrion 2008, 8(1):5-14.

31. Gray MW, Burger G, Lang BF: Mitochondrial evolution. Science 1999, 283(5407):1476-1481

32. Archibald JM, Richards TA: Gene transfer: anything goes in plant mitochondria. BMC Biol 2010, 8:147.

33. Bergthorsson $U$, Adams KL, Thomason B, Palmer JD: Widespread horizontal transfer of mitochondrial genes in flowering plants. Nature 2003, 424(6945):197-201

34. Thompson K: Cytoplasmic male sterility in oilseed rape. Heredity 1972, 29:253-257.

35. Shiga T, Baba S: Cytoplasmic male sterility in rape plants (Brassica napus L.). Jpn J Breed 1971, 21:16-17.

36. Fu T: Production and research of rapeseed in the People's Republic of China. Eucaroia Cruciferae Newsletter 1981, 6:6-7.

37. Arrieta-Montiel M, Lyznik A, Woloszynska M, Janska H, Tohme J, Mackenzie S: Tracing evolutionary and developmental implications of mitochondrial stoichiometric shifting in the common bean. Genetics 2001, 158(2):851-864

38. Janska H, Sarria R, Woloszynska M, Arrieta-Montiel M, Mackenzie SA: Stoichiometric shifts in the common bean mitochondrial genome leading to male sterility and spontaneous reversion to fertility. Plant Cell 1998, 10(7):1163-1180.

39. Burger G, Gray MW, Lang BF: Mitochondrial genomes: anything goes. Trends in genetics 2003, 19(12):709-716.

40. Bullerwell CE, Gray MW: Evolution of the mitochondrial genome: protist connections to animals, fungi and plants. Current opinion in microbiology 2004, 7(5):528-534.

41. Lang BF, Gray MW, Burger G: Mitochondrial genome evolution and the origin of eukaryotes. Annu Rev Genet 1999, 33:351-397.

42. Boore JL: Animal mitochondrial genomes. Nucleic Acids Res 1999, 27(8):1767-1780

43. Gray MW, Lang BF, Cedergren R, Golding GB, Lemieux C, Sankoff D, Turmel M, Brossard N, Delage E, Littlejohn TG, et al: Genome structure and gene content in protist mitochondrial DNAs. Nucleic Acids Res 1998 26(4):865-878

44. Schnable PS, Wise RP: The molecular basis of cytoplasmic male sterility and fertility restoration. Trends in Plant Science 1998, 3(5):175-180.

45. Altschul SF, Gish W, Miller W, Myers EW, Lipman DJ: Basic local alignment search tool. J Mol Biol 1990, 215(3):403-410.

46. Lowe TM, Eddy SR: tRNAscan-SE: a program for improved detection of transfer RNA genes in genomic sequence. Nucleic Acids Res 1997, 25(5):955-964.

47. Darling AE, Mau B, Perna NT: progressiveMauve: multiple genome alignment with gene gain, loss and rearrangement. PLoS One 2010, 5(6): e11147.

48. Benson G: Tandem repeats finder: a program to analyze DNA sequences. Nucleic Acids Res 1999, 27(2):573-580.

49. Murtagh F: Complexities of Hierarchic Clustering Algorithms: the state of the art. Computational Statistics Quarterly 1984, 1:101-113. doi:10.1186/1471-2164-12-497

Cite this article as: Chang et al:: Mitochondrial genome sequencing helps show the evolutionary mechanism of mitochondrial genome formation in Brassica. BMC Genomics 2011 12:497.

\section{Submit your next manuscript to BioMed Central and take full advantage of:}

- Convenient online submission

- Thorough peer review

- No space constraints or color figure charges

- Immediate publication on acceptance

- Inclusion in PubMed, CAS, Scopus and Google Scholar

- Research which is freely available for redistribution

Submit your manuscript at www.biomedcentral.com/submit
C Biomed Central 